\title{
Modelling and analysis of a high-speed turbine impeller concerning mistuning
}

\author{
Robby Weber ${ }^{1, *}$, Arnold Kühhorn ${ }^{1}$, and Christoph R. Heinrich ${ }^{1}$ \\ ${ }^{1}$ Chair of Structural Mechanics and Vehicle Vibration Technology \\ Brandenburg University of Technology Cottbus-Senftenberg \\ D-03046 Cottbus
}

\begin{abstract}
As-manufactured impellers behave significantly different from nominal impellers. There are no identical blades due to geometric and material deviations. In this paper three model updating procedures are discussed with the objective to achieve realistic models of as-manufactured impellers. The techniques are applied to radial inflow turbine wheel of an exhaust gas turbocharger. The first approach creates a model through optical measurement and mesh morphing. The second approach is based on a contactless measurement of blade individual vibration responses. An iterative update process gains the corresponding mistuning pattern and thus the associated model. Third, a model is found by an optimisation, that identified a mistuning pattern, that fits modal characteristics, which are evaluated during experimental modal analysis at vacuum. In-depth analyses of these models are realised to determine advantages and drawbacks of the procedures.
\end{abstract}

\section{Introduction}

Both, the geometrical deviation of the shape as a result of milling or casting process and the material inhomogeneities can cause structural imperfections. In turbomachinery, it is assumed, that there are no identical blades since these imperfections are unavoidable. In the blade integrated disk (blisk) technology, this induces localisation effects and vibration amplification. These effects are well-known as mistuning.

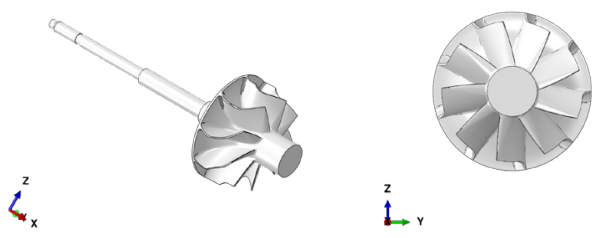

Figure 1. Object of investigation: high speed turbine impeller T040 INCO

\footnotetext{
*e-mail: robby.weber@b-tu.de
} 
Compared to the tuned reference, mistuning tends to increase the dynamic stress, so the High Cycle Fatigue life is generally reduced. Whitehead [1] developed a limit of forced response amplification, which is determined by the number of blades only. Meanwhile, less conservative limits were deduced[2, 3].

However, the evaluation of realistic forced response amplifications is a key issue for a safe design of turbomachinery [4]. Therefore the deviations between blades have to be taken into account. There are at least two fundamental ideas to consider mistuning. First, there are blade-individual material parameters applied during a Finite Element Simulation. Second, the mesh of an FE-Model is morphed to a point cloud, gained by an optical topography measurement.

In the scope of this paper, both types of modelling are demonstrated. The object of investigation is a high-speed turbine impeller, shown in Figure 1. Motivated by current publications [5-7] the process of optical measurement of the surface is presented briefly. Furthermore two methods, which vary model properties, are discussed. Then substantial modal parameters are compared with measurement results. Finally, the frequency response functions (FRF) of all models are compared to measurement results.

\section{Geometric optical measurement}

There is an ongoing development [5-8] of methods to derive a Finite Element Models of axial bladed structures by scanning their surfaces. Here an equivalent approach is shown. First, the surface is scanned (see Fig. 2 left). Thereby a point cloud of the impeller is generated. The optical system uses a blue-light fringe projector and two high-resolution cameras to allow a fast acquisition of a large number of points. The optical measurement is widely documented in the literature [5, 9].

The surface of the as-designed Finite Element Model is artificially matched to the optical scan data via an iterative closest point procedure. In fact, nodes are moved stepwise vertically towards their corresponding element surface. Figure 2 centre shows the initial shell surface and the point cloud data. Once the iteration process is finished, an as-manufactured model is developed (compare Fig. 2 right). Hence structural analysis can be executed.

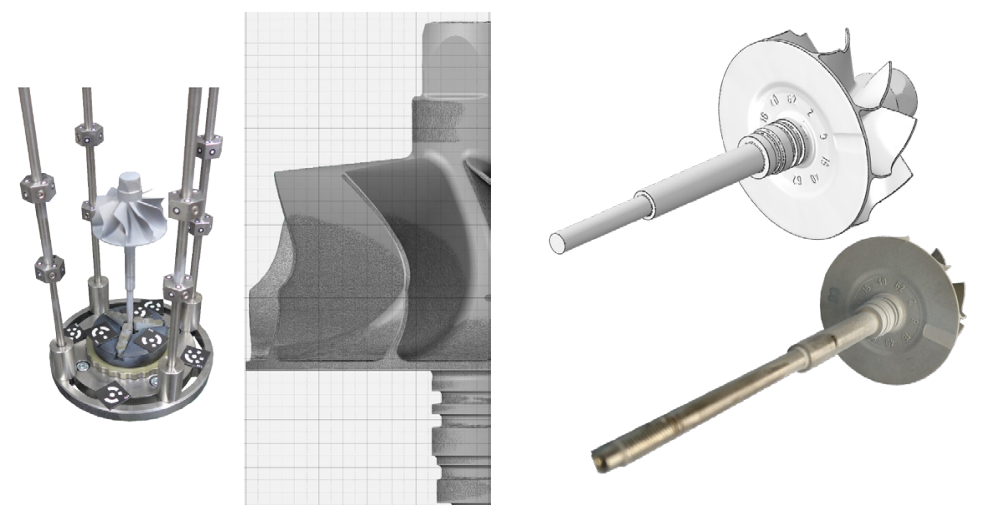

Figure 2. Steps of optical topography measurement: left: measurement setup, centre: meshmorphing and right: geometric updated FE model and manufactured impeller with marks 


\section{Experimental modal analysis}

Additionally, the object of investigation is analysed via blade vibration measurement. Therefore, the forced vibration of all blades is measured non-intrusively by a laser Doppler vibrometer (LDV). A piezoelectric element, located at the hub of the turbine, excites the vibration. Figure 3 left shows a picture of the impeller on top of a miniature table and Fig. 3 centre depicts the schematic measurement setup. Once the frequency response function (FRF) (see

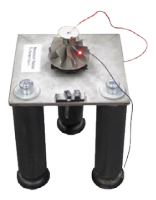

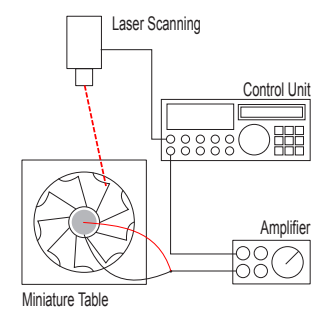

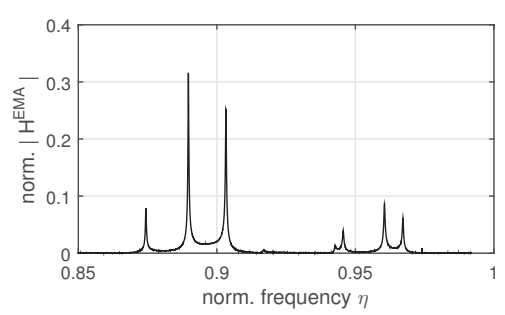

Figure 3. Experimental Modal Analyses of turbine impeller T040 INCO: left: measurement setup, centre: schematic visualisation and right: normalised forced response function of blade 4

Fig. 3 right) is obtained from all blades, modal parameters - as eigenfrequency, damping and mode shape - can be identified. Since the eigenfrequencies are in a close proximity a Multi-Degree of Freedom fitting approach has to be applied. Therein (see Ewins [10]), the differences between a measured FRF and their synthetic counterpart

$$
H_{k l}^{M D O F}(\Omega)=\sum_{m=1}^{M}\left[2 \frac{\omega_{m}\left(r_{k l m}^{r e} \zeta_{m}-r_{k l m}^{i m} \sqrt{1-\zeta_{m}^{2}}\right)+i \cdot r_{k l m}^{r e} \Omega}{\omega_{m}^{2}-\Omega^{2}+i \cdot 2 \zeta_{m} \omega_{m} \Omega}\right]
$$

is minimized within a frequency range of interest.

$$
\min \Delta=\min \left(\sum_{\eta=0.85}^{\eta=1.0}\left[H_{k l}^{M D O F}(\Omega(\eta))-H_{k l}^{E M A}(\Omega(\eta))\right]^{2}\right)
$$

Figure 4 compares the modeshapes and eigenfrequencies between the numerical modal analysis of the GOM-updated model and the experimental modal analysis (EMA). The Modal Assurance Criterion [11] is given in Fig. 4 left. The values are extremely poor. The dynamic behaviour of the experimentally measured structure does not correlate with the one predicted by the GOM-updated model. The eigenfrequencies (Fig. 4 right) indicate the same.
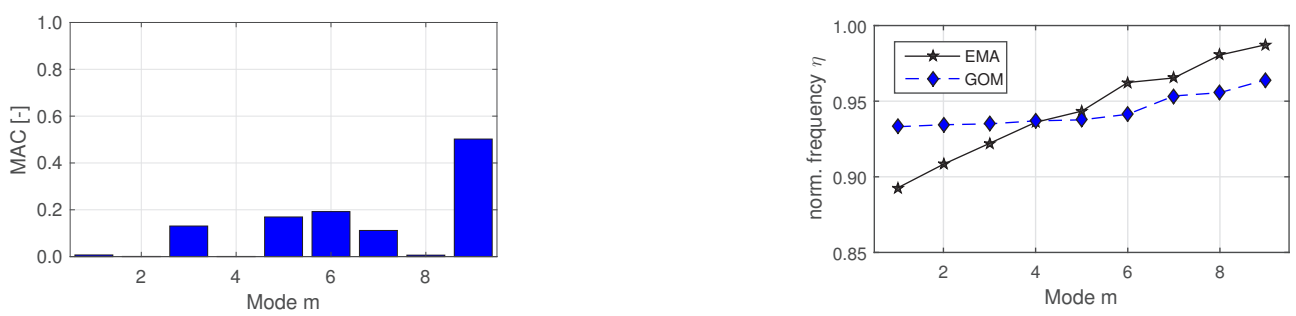

Figure 4. Comparison of modal properties: left: modal assurance criterion of eigenmodes and right: normalized frequencies between as-manufactured impeller and geometrical measured model 


\section{Experimental mistuning identification}

Maywald et al. [6] proposed an approach to experimentally determine blade-individual eigenfrequencies. In this method, a blisk is excited by a miniature modal hammer while the vibration response is measured non-intrusively by a laser vibrometer. Further details are given in US Patent [12]. The measurement setup has to be modified because the blades are too small to excite them with a miniature modal hammer. Instead a similar measurement step is used which is shown in Fig. 3. Here, small masses are placed to every non-measured blade (see Fig. 5 left and centre. Figure 5 right depicts the isolated eigenfrequencies of every blade. It is
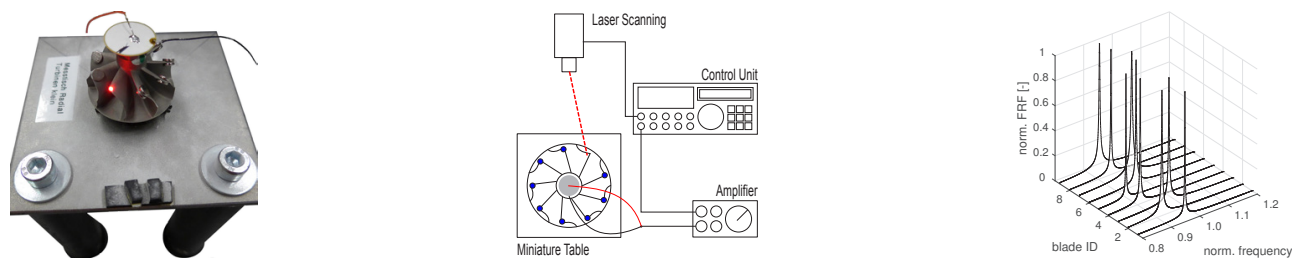

Figure 5. Blade-by-blade measurement: left: measurement setup, center: schematic visualisation and right: isolated eigenfrequencies

obvious, that they are unique. This complies with the assumption, that there are no identical blades. A mistuning pattern $\Delta \omega$ identifies deviations in eigenfrequencies of the first mode family of blade $k$

$$
\Delta \omega_{1}=\frac{\omega_{k, 1}-\bar{\omega}_{1}}{\bar{\omega}_{1}}
$$

\section{Inverse mistuning identification method}

The authors [13] propose another method to determine the mistuning pattern. In this approach the mistuning pattern is estimated by an elitist genetic algorithm (a variant of NSGA-II)[14], provided by MATLAB. The objective of this optimisation is to match modal parameters,

$$
\begin{array}{lc}
\min _{c} & \left\{\epsilon_{f}+\epsilon_{\Phi}\right\} \\
\text { s.t. } & \left|c_{k}\right| \quad \leq 10 \% \quad \forall k \in N_{\text {Blades }}
\end{array}
$$

identified by an experimental modal analysis as shown in Fig. 3, but conducted under vacuum condition. A reduced order model [15] is used to minimise computational costs. The basic idea was initially proposed by Feiner and Griffin $[16,17]$ in 2003. They introduced another reduced order model and focussed on axial bladed rotors.
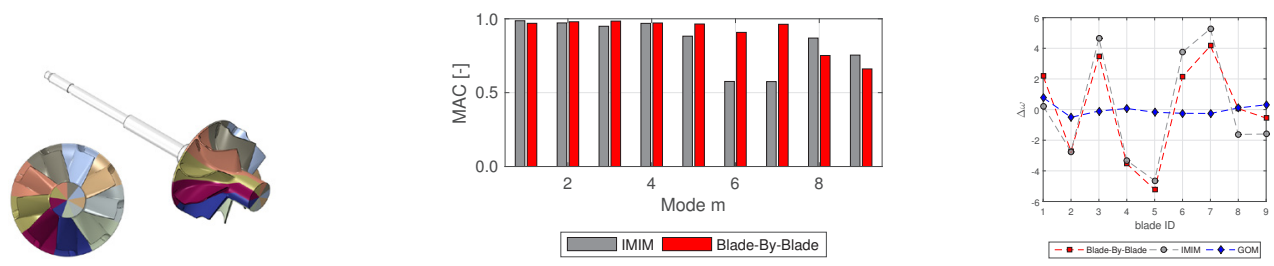

Figure 6. Left: blade individual material properties, centre: MAC values of material property updated FE model and right: blade individual eigenfrequencies 


\section{Assessment}

The experimentally identified mistuning pattern $\Delta \omega$ and the mistuning parameters $c_{k}$, which are used to introduce mistuning to the reduced order model, are blade-individual parameters that can be interpreted as a variation of material properties. Therefore every blade sector is assigned a unique Young's modulus (see Fig. 6 left). Figure 6 right compares blade-individual eigenfrequencies, which are measured during the experimental mistuning identification or which are derived from the FE model. There is a distinct similarity between the BladeBy-Blade pattern, which represents the experimental method, and the IMIM, which denotes the inverse identification. Additionally, there is the third pattern (blue line), that shows a significantly lower level of mistuning since the eigenfrequencies are close to each other. The MAC values of the IMIM, as well as the Blade-By-Blade and the measured data, are close to 1 (see Fig. 6 centre), which indicates an excellent model update.
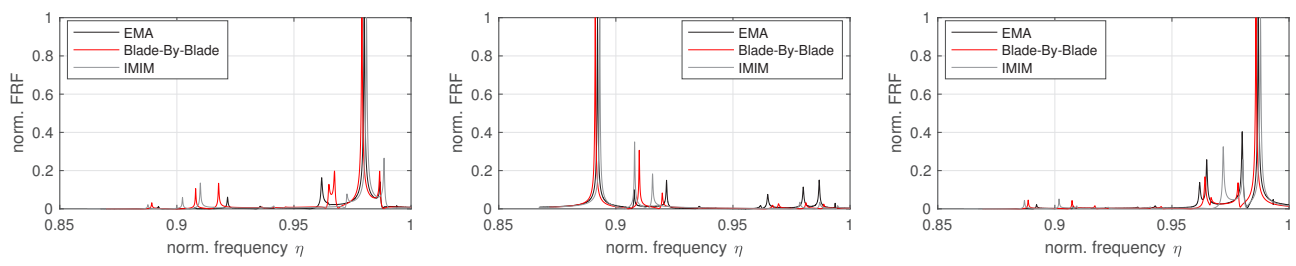

Figure 7. Frequency response function: left: Blade 3, center: Blade 5 and right: Blade 7

However, the Frequency Response Functions shown in Fig. 7 confirm, that at least the high responding modes in every blade individual dynamic are predicted very well.

\section{Conclusion}

In this paper, three approaches to model mistuning are shown. First, the Finite Element Model based on a geometric surface scan is not able to predict the dynamic behaviour of the mistuned structure. Hemberger et al. [18] show that material inhomogeneities are the primary source of mistuning considering small high-speed impellers. In addition, the level of random and systematic error [9] of geometric optical measurements may be too high to evaluate geometric mistuning. Second, an experimental mistuning identification technique is shown, which is able to predict basic dynamic characteristics. Third, an identification method, which relies purely on (reduced) FE calculations to match the measured modal parameters, is capable of simulating a Frequency Response Function similar to the measured data.

The discussed techniques are applied to measurement data, which are obtained from a separate component (lab testing) at rest. Only the Inverse Mistuning Identification Method is feasible under operational conditions. However, the modal properties have to be identified based on tip timing or strain gauge measurement, which may be faulty. According to the authors' point of view, a instrumentation with strain gauges will inevitably alter the object under investigation by changing the mass and the stiffness of the system. Therefore a direct contact vibration measurement by accelerometers is not recommendable.

The work presented has been supported by the Research Association for Combustion Engines (FVV). The authors thank for this commitment. The independent investigations contribute to the research project Mistuning and Damping of Centrifugal Compressors and Turbines funded by FVV capital (FVV 1229). Further thanks are given to the working group of industrial representatives. 


\section{References}

[1] D. S. Whitehead, Journal of Mechanical Engineering Science, 8, pp. 15-21, (1966)

[2] C. Martel and R. Corral, Journal of Eng for Gas Turb and Power, 131, pp. 1-10, (2009)

[3] F. Figaschewsky and A. Kühhorn, Proceedings of ASME Turbo Expo, GT2015-43121, (2015)

[4] H. Schönenborn, U. Retze, G. Ziller and P. Waniczek, Proceedings of ASME Turbo Expo, GT2010-22447, (2010)

[5] A. A. Kaszynski, J. A. Beck and J. M. Brown, Proceedings of ASME Turbo Expo, GT2014-26925, (2014)

[6] T. Maywald, T. Backhaus, S. Schrape and A. Kühhorn, Proceedings of ASME Turbo Expo, GT2017-63446, (2017)

[7] D. L. Gillaugh, A. A. Kaszynski, J. M. Brown, J. A. Beck and J. C. Slater, Proceedings of ASME Turbo Expo, GT2018-76888, (2018)

[8] A. A. Kaszynski, J. A. Beck and J. M. Brown, Journal of Eng for Gas Turb and Power, 135, pp. 102504, (2013)

[9] T. Backhaus, T. Maywald, S. Schrape, M. Voigt, R. Mailach, Proceedings of ASME Turbo Expo, GT2017-64243, (2017)

[10] D. J. Ewins, "Modal Testing: Theory, Practice and Application.", 2. Edition. Engineering Dynamics Series. Baldock et al.: Research Studies Press LTD, (2000)

[11] R. J. Allemang, Journal of Sound and Vibration, 37(8), pp. 14-23, (2003)

[12] A. Kühhorn, B. Beirow, "Method for Determining Blade Mistuning on Integrally Manufactured Rotor Wheels", Patent US 2010/0286934 A1, (2010)

[13] R. Weber, A. Kühhorn, Proceedings of ASME Turbo Expo, GT2018-75382, (2018)

[14] K. Deb, "Multi-Objective Optimization Using Evolutionary Alogorithms", John Wiley $\mathcal{E}$ Sons, (2001)

[15] M. T. Yang, J. H. Griffin, Journal of Eng for Gas Turb and Power, 123, pp. 893-900, (2001)

[16] D. M. Feiner, J. H. Griffin, Journal of Turbomachinery, 126, pp. 150-158 (2003)

[17] D. M. Feiner, J. H. Griffin, Journal of Turbomachinery, 126, pp. 159-165 (2003)

[18] D. Hemberger, D. Filsinger, H.-J. Bauer, Proceedings of ASME Turbo Expo, GT201425099, (2014) 